\title{
Processamento Visual da Forma: Evidências para Canais Múltiplos de Freqüências Angulares em Humanos
}

\author{
Natanael Antonio dos Santos ${ }^{12}$ \\ Universidade Federal da Paraíba \\ Renata Maria Toscano Barreto Lyra Nogueira \\ Maria Lúcia de Bustamante Simas \\ Universidade Federal de Pernambuco
}

\begin{abstract}
Resumo
O objetivo deste trabalho foi caracterizar e comparar curvas de resposta do sistema visual humano para filtros de freqüências angulares de 1 e 4 ciclos $/ 360^{\circ}$ em coordenadas polares. Mensuramos 15 curvas para cada filtro com o paradigma de somação de resposta de supralimiar e com a participação de 5 voluntários adultos. Os resultados demonstraram somações máximas de limiar de contraste na freqüência de teste dos filtros angulares $\left(1 \mathrm{e} 4\right.$ ciclos $\left./ 360^{\circ}\right)$ circundadas por inibições nas freqüências vizinhas às freqüências de teste de cada filtro. Estes resultados são consistentes com a existência de canais ou filtros angulares de banda estreita operando no sistema visual humano através do processo de somação ou inibição em faixa de freqüências específicas.

Palavras-chave: Processamento visual da forma; freqüência angular; filtro de banda-estreita; paradigma de somação de supralimiar.
\end{abstract}

Visual Processing of Form: Evidences for Angular Narrow-band Frequency Channels in Humans

\begin{abstract}
The aim of this work was to characterize and to compare the response curves of the human visual system for angular filters of 1 and 4 cycles/ $360^{\circ}$ defined in polar coordinates. We measured 15 curves for each filter with 5 adult volunteers using a supra-threshold summation method with a forced-choice paradigm. The results showed maximum summation effects at test frequencies for angular $\left(1\right.$ and 4 cycles $\left./ 360^{\circ}\right)$ filters surrounded on both sides by strong inhibition. These results were in agreement with the existence of channels or narrow-band angular filters operating in the human visual system either through summation or inhibition of specific frequency ranges.

Keywords: Visual processing of form; angular frequency; narrow-band frequency filters; supra-threshold summation method.
\end{abstract}

O reconhecimento de cenas visuais ou de objetos é um processo fundamental na percepção ou no processamento visual da forma. A idéia da decomposição de objetos complexos em subunidades simples ou elementares e o fato de unidades ou células nervosas responderem apenas a determinadas características dos objetos levaram vários pesquisadores a caracterizar a resposta do sistema visual para estímulos elementares com o objetivo de tentar identificar os atributos ou as subunidades nas quais um padrão complexo é decomposto e processado. Neste contexto, sugiram modelos teóricos ligados à percepção visual da forma, que investigam como o sistema visual humano processa informações espaciais ou objetos, por exemplo, o modelo de canais múltiplos. Informações mais detalhadas sobre alguns modelos ligados a percepção visual da forma podem ser encontrado em Santos e Simas (2001).

A idéia de canais múltiplos de freqüências espaciais foi proposta inicialmente por Campbell e Robson (1968). Essa abordagem assume que o sistema visual humano responde seletivamente a freqüências espaciais, ou seja, o sistema visual processa simultaneamente diferentes faixas estreitas de freqüências espaciais (Blakemore \& Campbell, 1969; Campbell \& Robson, 1968; Sachs, Nachmias \& Robson, 1971). Em outras palavras, o modelo de canais múltiplos que se fundamenta

\footnotetext{
${ }^{1}$ Endereço para correspondência: Departamento de Psicologia, Centro de Ciências Humanas Letras e Artes, UFPB. Campus I, Cidade Universitária, Cj Castelo Branco, 58051 900, João Pessoa, PB. Fone: (83)2354439; Fax: (83)2167064. E-mail: natanael@cchla.ufpb.br\&natanael_labv@bol.com.br

${ }^{2}$ Agradecemos o apoio financeiro do CNPq e a todos os participantes da pesquisa.
}

na análise de sistema linear defende que a percepção de um objeto (ou cena visual) qualquer ocorre porque o nosso sistema visual executa uma análise ou decomposição do objeto em seus componentes mais elementares, uma espécie de análise de Fourier (ver Blakemore \& Campbell, 1969; De Valois \& De Valois, 1988).

As primeiras evidências psicofísicas para canais múltiplos de freqüências espaciais vieram a partir do surgimento da técnica da adaptação seletiva a freqüências espaciais. Essa técnica surgiu de um trabalho de Gilinski (1968) que observou que depois de visualizar um padrão listrado de contraste alto por um período de tempo, observadores podem mostrar um prejuízo para perceber padrões de freqüência e orientação idênticas, mas de contraste mais baixo. Esse fenômeno descrito por Gilinski se tornou conhecido como adaptação e foi observado ser altamente seletivo para orientação e freqüência espacial (Blakemore \& Campbell, 1969; De Valois \& De Valois, 1988; Menees, 1998).

Um dos primeiros e mais convincentes estudos realizados com o método da adaptação seletiva favoráveis à existência de canais múltiplos de freqüências espaciais foi realizado por Blakemore e Campbell (1969). Esses autores verificaram que a adaptação à grade senoidal eleva o limiar da grade senoidal próxima à freqüência adaptada.

Atualmente, estudos psicofísicos em humanos e estudos neurofisiológicos em macacos e gatos fornecem fortes evidências para o modelo de canais múltiplos ou mecanismos sintonizados para banda estreita de freqüências espaciais (Blakemore \& Campbell, 1969; De Valois \& De Valois, 1988; Dakin \& Hess, 1997; Maffei \& 
Fiorentini, 1973; Menees, 1998; Olds \& Engel, 1998; Vuilleumier, Armony, Driver \& Dolan, 2003; Wilson, Levi, Maffei, Rovamo \& De Valois, 1990). Entretanto, a maioria absoluta destes utiliza grade senoidal vertical em coordenadas cartesianas para caracterizar a existência de filtros ou canais sintonizados para freqüências espaciais. Do ponto de vista psicofísico, um canal ou um filtro corresponde a uma estrutura visual independente que lida com uma faixa estreita de freqüência espacial. Freqüência espacial é o número de ciclos (ou listras claras e escuras) por grau de ângulo visual (cpg).

Nas últimas décadas, alguns pesquisadores procuraram estabelecer a existência de mecanismos ou canais múltiplos para estímulos diferentes de grade senoidal, por exemplo, padrões radiais (Santos \& Simas, 2002) e angulares em coordenadas polares (Simas \& Santos, 2002a). Simas e colaboradores mensuraram a resposta do sistema visual humano para vários filtros de freqüências angulares com o método psicofísico de somação de resposta de supralimiar aliado ao procedimento da escolha forçada (Simas \& Dodwell, 1990; Simas, Frutuoso \& Vieira, 1992; Simas \& Santos, 2002a, 2002b). Os resultados encontrados demonstraram efeitos de somação absoluta e relativa nas freqüências angulares de teste dos filtros circundadas por inibições. O que levou Simas e colaboradores a concluírem a favor da existência de alguma seletividade para faixas específicas de freqüências angulares. Santos e Simas (2002) mensuram curvas de resposta ao contraste para os filtros de freqüências radiais de 1 e $4 \mathrm{cpg}$ em coordenadas polares e comparam com os filtros de freqüências espaciais do tipo grade senoidal vertical de 1 e 4 cpg em coordenadas cartesianas. $\mathrm{O}$ objetivo principal foi estabelecer a existência de filtros de freqüências radiais comparados a filtros de freqüências espaciais do tipo grade senoidal vertical. As estimativas também foram realizadas com o paradigma de somação de resposta de supralimiar. Os resultados foram consistentes com a existência de filtros de freqüências radiais de banda-estreita em coordenadas polares.

\section{Evidências neurofisiológicas para estímulos radiais e angulares em coordenadas polares}

Existem evidências, baseadas em estudos psicofísicos e neurofisiológicos, de que o processamento linear ou filtragem espacial local sintonizada para orientação e freqüência espacial de estímulos projetados na retina ocorre nos estágios iniciais da visão, isto é, em neurônios do córtex visual primário, V1 (De Valois \& De Valois, 1988; Manahilov \& Simpson, 2001; Wilkinson \& cols., 2000; Wilson \& Wilkinson, 1998; Wilson, Wilkinson \& Asaad, 1997). Por outro lado, até o momento não existe nenhum relato que unidades neurais da área visual primária, V1, executem filtragem de estímulos em coordenadas polares, amostrando áreas grandes do campo visual. Neste contexto, resultados psicofísicos em humanos (Wilkinson, Wilson \& Habak, 1998; Wilson \& Wilkinson, 1997, 1998; Wilson \& cols, 1997), neurofisiológicos em primatas (Bruce, Desimone \& Gross, 1981; Desimone, 1991; Desimone \& Schein, 1987, Gallant, Connor, Rakshit, Lewis \& van Essen 1996; Hegde \& Van Essen, 2000) e imagem de ressonância magnética funcional em humanos (Wilkinson \& cols., 2000) destacam que o processamento da informação que ocorre em vias intermediárias (Ex.: área visual V4) e áreas mais avançadas (Ex.: córtex visual ínfero-temporal, IT) do sistema visual são modeladas por filtros espaciais globais em coordenadas polares. Alguns desses trabalhos destacam que a área V4 pode formar o principal estágio intermediário da visão da forma de V1 para IT (Heywood, Gadotti \& Cowey, 1992; Merigan, 1996; van Essen, Anderson \& Felleman, 1992; Wilkinson \& cols., 2000; Young, 1992). Assim, é possível que a filtragem e integração de padrões radiais e angulares em coordenadas polares ocorram em áreas visuais extraestriatais (Ex.: V4 e IT). O estudo de Willkinson e colaboradores (2000) com a técnica de imagem de ressonância magnética funcional em humanos reforçou os estudos psicofísicos com humanos e neurofisiológicos com primatas que demonstraram inicialmente que estímulos em coordenadas polares são processados em áreas visuais extra-estriatais (Ex.: V4 e IT).

O presente estudo visou dar continuidade aos experimentos que investigam o processamento visual humano de estímulos em coordenadas polares (Ex.: freqüências radiais e angulares). Neste sentido, este trabalho procurou caracterizar mecanismos ou canais sintonizados para freqüências angulares com bandas de passagem centradas em 1 e 4 ciclos $/ 360^{\circ}$. O objetivo principal foi caracterizar a existência de canais de banda-estreita para freqüências angulares definidos em coordenadas polares, comparados a filtros de freqüências espaciais com grade senoidal em coordenadas cartesianas, que são bem estabelecidos na literatura. Os filtros escolhidos possibilitam uma comparação geral entre as curvas de filtragem destes padrões. Os filtros foram mensurados com o método psicofísico de somação de resposta de supralimiar aliado ao método psicofísico da escolha forçada (Santos \& Simas, 2002; Simas \& Dodwell, 1990; Simas \& cols., 1992; Simas \& Santos, 2002a, 2002b). Estudos desta natureza são raros e pouco estabelecidos na literatura.

Um dos pressupostos básicos destes experimentos é que estímulos de freqüência radial, freqüência angular e grade senoidal são processados por vias e áreas visuais diferentes. Em outras palavras, acreditamos na possibilidade das áreas superiores do sistema visual (Ex.: V4 e IT) processarem informação visual da forma em termos de freqüências radiais e angulares em coordenadas polares, o que pode envolver filtragem de faixas ou áreas globais do campo visual.

Experimentos com o paradigma de somação de resposta de supralimiar utilizam níveis de contraste supralimiares para mensurar o máximo da função, isto é, a freqüência de teste é somada pixel a pixel às freqüências de fundo que são apresentadas a alto contraste. Nesse, enquanto o contraste da freqüência de teste é variado de acordo com a sensibilidade do voluntário, o contraste da freqüência de fundo é fixo acima do limiar (i.e., supraliminar). Desta forma, se um estímulo contendo apenas uma das freqüências de fundo é comparado a um estímulo em que a mesma freqüência de fundo é somada a freqüência de teste, a única forma de diferenciar entre os dois estímulos é detectar a presença da freqüência de teste em um destes. Logo, durante o processo de filtragem, três tipos de resposta ou efeito podem ocorrer: 1) se a superposição da freqüência de teste do filtro à freqüência de fundo facilita ou diminui o valor de contraste absoluto necessário à detecção, isto pode ser porque a freqüência de teste e a freqüência de 
fundo estão sendo processadas pelo mesmo mecanismo ou canal (efeito de somação); 2) se a superposição da freqüência de teste à freqüência de fundo dificulta ou aumenta o valor de contraste absoluto, isto podem ser porque elas são processadas por canais distintos que interagem entre si possivelmente de forma antagônica (efeito inibitório); e 3) se a superposição da freqüência de teste à freqüência de fundo não facilita e nem dificulta o valor de contraste absoluto (efeito independente), a freqüência de teste e a freqüência de fundo possivelmente estão sendo processadas por canais distintos que não interagem entre si. A sensibilidade ao contraste da freqüência de teste em função das freqüências de fundo, usando como referência o limiar absoluto de detecção da freqüência de teste, indicará a independência dos mecanismos de detecção ou os efeitos de interdependência que se caracterizam como somação ou inibição. Informações mais detalhadas sobre este paradigma podem ser encontrado em Simas e colaboradores (Santos \& Simas, 2002; Simas \& Dodwell, 1990; Simas \& cols., 1992; Simas \& Santos, 2002a).

\section{Método}

\section{Participantes}

Participaram dos experimentos 5 adultos de ambos os sexos na faixa etária de 20-26 anos com acuidade visual normal ou corrigida.

\section{Equipamentos e Estímulos}

Foi utilizado um sistema desenvolvido no próprio laboratório, que compreende um monitor de vídeo SONYBVM-1910, com entrada "RGBsync" entrelaçada e interfaciado a um microcomputador 486 de $30 \mathrm{MHz}$ através de um placa de aquisição Data-Translation DT-2853. Um programa escrito em linguagem $\mathrm{C}$ foi desenvolvido no próprio laboratório para executar os experimentos. Uma cadeira giratória foi fixada a 150 $\mathrm{cm}$ da tela do monitor de vídeo. Uma tábua cinza foi situada acima do monitor de vídeo para onde o voluntário, entre uma apresentação e outra, deveria fixar o olhar com o objetivo de diminuir os efeitos de pós-imagem. A luminância média foi de $6,9 \mathrm{~cd} / \mathrm{m}^{2}$ ajustada por um fotômetro do tipo SPOT MET'TER, com precisão de um grau, ASAHI PENTAX. A luminância mínima foi de $6,2 \mathrm{~cd} / \mathrm{m}^{2}$ e a máxima de $7,5 \mathrm{~cd} / \mathrm{m}^{2}$. O ambiente do laboratório era cinza para melhor controle da luminância.

Todos os estímulos foram gerados em tons de cinza, circulares, com diâmetros de 7,25 graus de ângulo visual a $150 \mathrm{~cm}$ de distância da tela (ver Figura 1) e eram apresentados em tempo real no monitor.

As estimativas dos filtros angulares de 1 e 4 ciclos $/ 360^{\circ}$ foram realizadas com pares de estímulos, estímulo de teste e estímulo de fundo. $\mathrm{O}$ estímulo de teste era composto pela freqüência angular de teste do filtro $\left(1\right.$ ou 4 ciclos $/ 360^{\circ}$ ) somada a uma das freqüências de fundos: $1 ; 2 ; 3 ; 4 ; 5 ; 6 ; 7 ; 8 ; 9 ; 10 ; 12 ; 16 ; 24 ; 32 ; 48 ; 64$ e 96 ciclos $/ 360^{\circ}$ (Figura 1). O estímulo de fundo para ambos os filtros angulares foi a própria freqüência de fundo angular sozinha. A Figura 1 ilustra exemplos de pares de estímulos de freqüências angulares. Outras informações sobre estes estímulos podem ser encontradas em Simas e Santos (2002a).

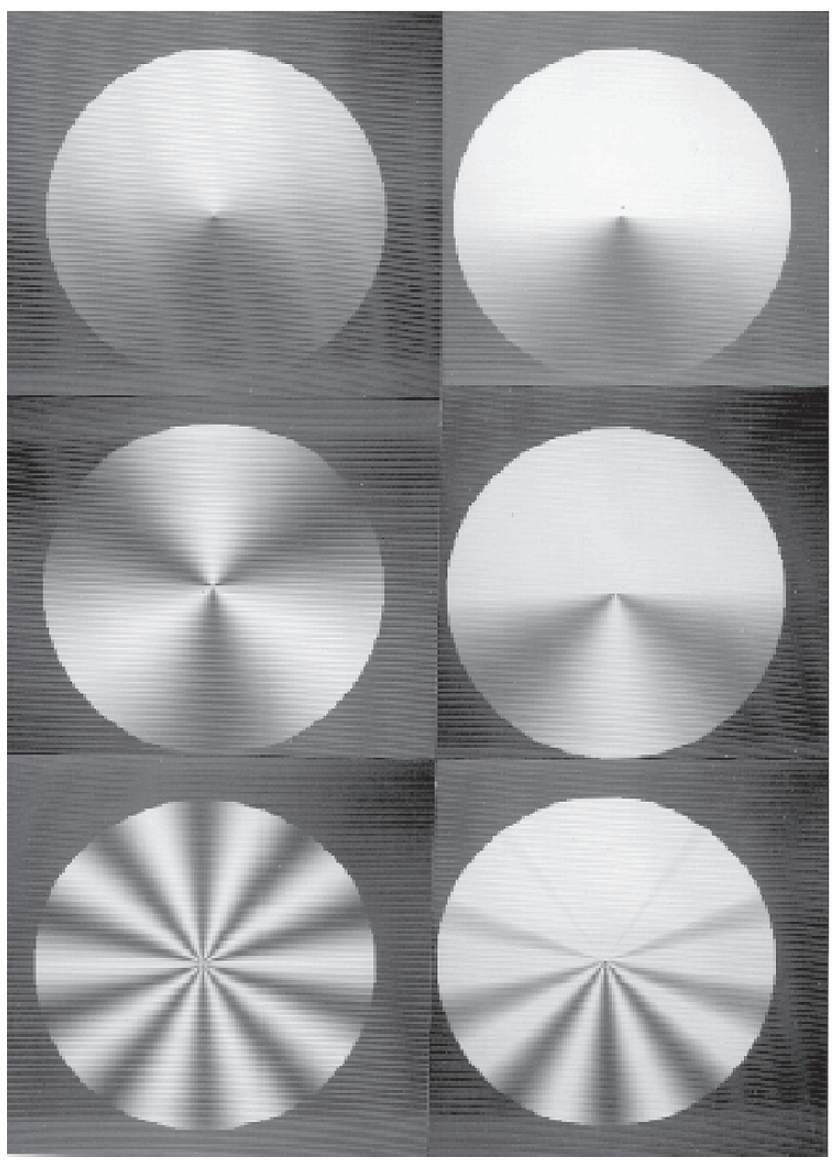

Figura 1. Exemplos de pares de estímulos de freqüências angulares utilizados para estimar o filtro angular de 1 ciclo $/ 360^{\circ}$. Na coluna da esquerda, de cima para baixo, as freqüências de fundo de 1, 3 e 10 ciclos $/ 360^{\circ}$ e na coluna da direta, as freqüências da esquerda (1, 3 e 10 ciclos) somadas a freqüência de teste do filtro (1 ciclo). Estímulos originalmente calibrados para serem vistos a $150 \mathrm{~cm}$ de distância antes de fotografados.

\section{Procedimento}

O procedimento de resposta de somação de supralimiar para cada filtro consistiu na apresentação sucessiva simples do par de estímulos e o voluntário teria que escolher dentre os dois estímulos qual destes continham a freqüência de teste do filtro angular somada à freqüência de fundo angular. $\mathrm{O}$ contraste da freqüência de fundo foi mantido constante e correspondia a cinco vezes o limiar absoluto de detecção da freqüência de teste do filtro de 1 ou 4 ciclos $/ 360^{\circ}$, tanto no estímulo de teste como no estímulo de fundo. Somente o contraste supralimiar da freqüência de teste era variada, diminuindo ou aumentando de acordo com o método psicofísico da escolha forçada (Simas \& Santos, 2002b; Wetherill \& Levitt, 1965). O critério adotado para variar o contraste da freqüência de teste era o de três acertos consecutivos para decrescer uma unidade e um erro para acrescer da mesma unidade $(0,8 \%)$. Este procedimento experimental se baseia no cálculo da probabilidade de acertos consecutivos por parte do participante, ou seja, em cerca de 100-150 apresentações de 
escolhas entre os dois estímulos, o estímulo de teste é percebido $79 \%$ das vezes pelo participante.

O experimento foi iniciado com um sinal sonoro seguido imediatamente pela apresentação do primeiro estímulo por 2s, seguido de um intervalo entre estímulos de 1s, seguido pela apresentação do segundo estímulo por $2 \mathrm{~s}$ e da resposta do participante. A ordem de apresentação dos estímulos era aleatória. Se a resposta do participante fosse correta, ela era seguida por outro sinal sonoro e um intervalo de 3 s para a seqüência temporal se repetir. Em outras palavras, 3s era o intervalo entre tentativas independente da resposta (ou escolha) ser correta ou não. O sinal sonoro que indicava o início da apresentação do par de estímulos e o que indicava a escolha correta eram diferentes e discretos. A sessão experimental variava em duração dependendo dos erros e acertos do voluntário até proporcionarem um total de 10 máximos e 10 mínimos conforme requerido para o final automático da mesma. No geral, sua duração era da ordem de 10-20 min, o que correspondia em média a 100 apresentações do par de estímulos.

Os participantes foram orientados antes da sessão a pressionar a barra de espaço quando julgassem que o estímulo de teste tivesse sido apresentado primeiro e qualquer tecla acima da barra de espaço quando julgasse que o mesmo tivesse sido apresentado em segundo lugar, isto é, após o estímulo de fundo.

A ordem de mensuração das freqüências angulares para cada curva foi aleatorizada por sorteio pelo participante. A curva de filtro angular foi composta por 17 freqüências. Cada freqüência foi medida pelo menos três vezes, em dias diferentes, por cada um dos 5 voluntários, totalizando 15 curvas (três curvas por voluntário) para cada filtro gerando uma amostra de 300 valores para cada uma das freqüências estimadas. Todas as estimativas foram feitas à distância de $150 \mathrm{~cm}$, com visão binocular.

\section{Resultados}

As freqüências angulares de fundo são apresentadas nos gráficos em função da quantidade de contraste necessário para identificar a presença da freqüência de teste do filtro de 1 e 4 ciclos $/ 360^{\circ}$ no par que continha a soma. Estamos chamando este dado de limiar para a freqüência de teste somada a cada freqüência de fundo de limiar de contraste para o estímulo teste na soma. A linha horizontal representa a linha de base, que equivale ao limiar absoluto para detectar a freqüência de teste dos filtros angulares de 1 e 4 ciclos $/ 360^{\circ}$ isoladas.

Nosso tratamento estatístico foi estimar o erro-padrão da média para cada distribuição de 300 valores mensurados para cada ponto e corrigidos para o tamanho da amostra pelo estatístico $t$-Student para obter o nível de confiança de 99\%. Em experimentos anteriores, foi estabelecido que o erro-padrão da média, corrigido pelo tamanho da amostra representando intervalo de confiança de $99 \%$ pelo estatístico $t$-Student, de acordo com o número de valores mensurados, é um critério mais estrito do que a utilização da ANOVA ou teste $t$ para amostras correlacionadas. Por exemplo, quando os intervalos dos errospadrões da média assim corrigidos se superpõem até mesmo pela metade, ainda assim um teste $t$-Student para amostras correlacionadas revela diferenças entre as médias que são significativas $\operatorname{com} p<0,05$. Nos casos em que os erros barras não se sobrepõem, as médias são significativas com $p<0,001$. A ANOVA tende a mostrar interações e efeitos significativos em todos fatores e não acrescenta muita informação sobre a tendência das curvas.
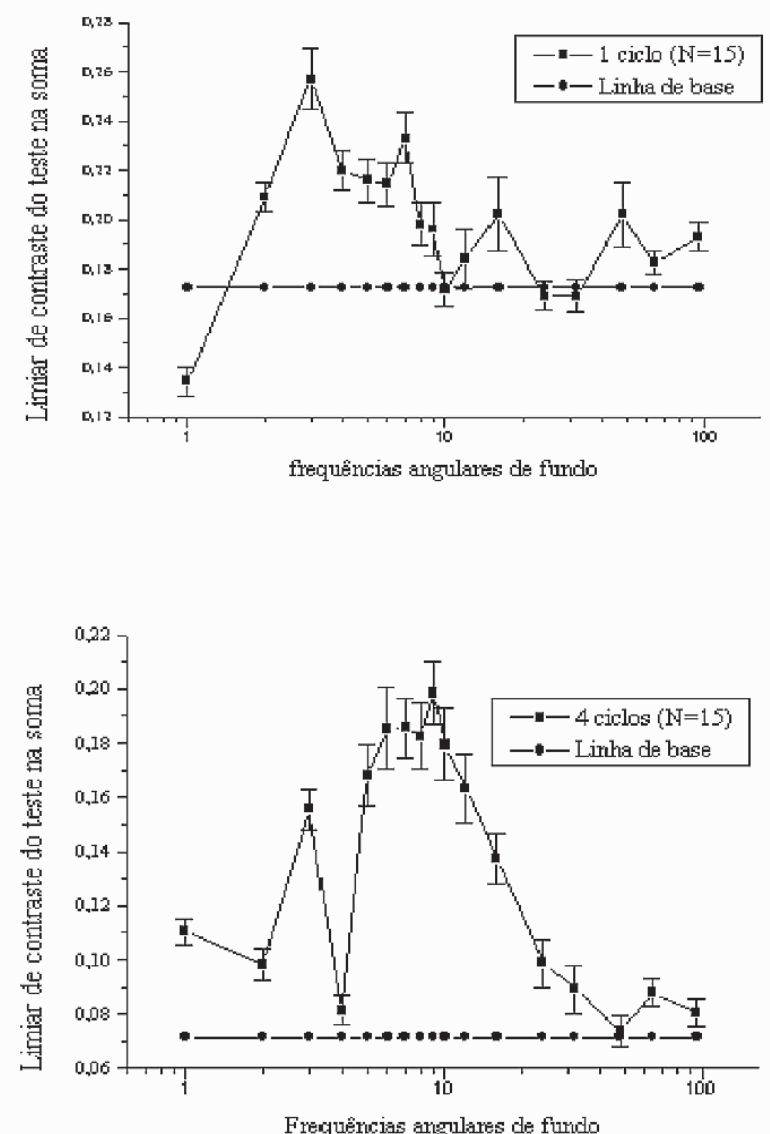

Figura 2. Mostra as curvas de resposta ao contraste da freqüência angular de teste do filtro de 1 ciclo acima e 4 ciclos abaixo somada às respectivas freqüências angulares de fundo representadas na abscissa.

A Figura 2 mostra as curvas de resposta ao contraste para os filtros de freqüências angulares de 1 e 4 ciclos $/ 360^{\circ}$, respectivamente. Nesta, os filtros de freqüências angulares de 1 e 4 ciclos $/ 360^{\circ}$ se comportam de maneira semelhante, isto é, eles apresentam efeito de somação máxima em suas respectivas freqüências angulares de teste $\left(1\right.$ e 4 ciclos $\left./ 360^{\circ}\right)$ circundadas de inibição nas freqüências vizinhas às freqüências angulares de teste (Figura 2). As razões entre somação e inibição máximas foram da ordem de 1,5 e 2,1 para os filtros de 1 e 4 ciclos $/ 360^{\circ}$, respectivamente. Estas razões foram encontradas nas freqüências vizinhas (Ex.: 2 ciclos no caso do filtro angular de 1 ciclo e 5 ciclos no caso do filtro angular de 4 ciclos) indicando claramente efeitos de somação ou filtragem.

\section{Discussão}

Estes resultados mostram que o efeito de somação máxima se deu na própria freqüência de teste dos filtros angulares, ladeado por 
inibição nas freqüências vizinhas (Figura 2). Observa-se, ainda, que a banda de passagem dos filtros mensurados neste trabalho é bem definida e apresenta alta seletividade. Estes resultados sugerem a existência de filtros de banda estreita seletivos para freqüências angulares nas faixas de freqüências angulares estudadas.

A noção de canal ou filtro supõe que um estímulo apresentado no campo visual não estimula todos os neurônios corticais que recebem informações daquela área da retina, mas apenas um grupo de neurônios seletivos para uma dimensão do estímulo, por exemplo, freqüência espacial. Assim, se um segundo estímulo que difere do primeiro ao longo da faixa de freqüência (ou uma outra dimensão qualquer) é apresentado na mesma área, ele ativará um grupo de neurônios diferente do primeiro. Em outras palavras, estímulos diferentes são processados por grupos ou subunidades neuroniais diferentes, os quais formam a base dos canais ou filtros de freqüências espaciais.

A largura de banda de um filtro se refere à função de resposta para as freqüências espaciais desse filtro. Dentro desse contexto, esperase que a curva de resposta para um filtro transmita informações 1) sobre a banda (ou bandas) de passagem do filtro; 2) sobre as freqüências em que o filtro responde menos ou não responde (deixa passar alguma coisa ou não deixa passar nada); e 3) sobre aquelas freqüências que estão fora da faixa de resposta do filtro. É importante destacar que, quanto mais estreita a largura da banda, mais alta sua seletividade à freqüência espacial e vice-versa.

A existência de filtros de freqüência espacial de banda estreita no sistema visual humano foi demonstrado inicialmente com estudos psicofísicos para estímulos cartesianos verticais, grade senoidal, (Blakemore \& Campbell, 1969; Graham, 1972; Sachs \& cols., 1971). Esses trabalhos iniciais indicaram que os canais que processavam banda estreita eram independentes. Entretanto, com o passar dos anos, novos estudos foram surgindo e verificou-se que esses canais podiam interagir entre si (Braddick, Campbell \& Atkinson, 1978; De Valois, 1977; Pollen \& Ronner, 1982; Tolhurst, 1972; Tolhurst \& Barfield, 1978). Em outras palavras, um modelo de canais múltiplos não implica necessariamente que em qualquer condição de estimulação e teste o sistema visual se comporte como completamente formado por canais de freqüências espaciais fortemente independentes (Sekuler, 1974).

Os nossos resultados com filtros de freqüências angulares de 1 e 4 ciclos $/ 360^{\circ}$ (Figura 2), também, demonstram efeitos de somação secundários em freqüências angulares de fundo mais baixas e mais altas. Por exemplo, o filtro angular de 1 ciclo $/ 360^{\circ}$ mostra efeito de somação relativa em freqüências mais altas (Ex.: 10,24 e 32 ciclos $\left(360^{\circ}\right)$, enquanto o filtro angular de 4 ciclos/ $360^{\circ}$ mostra efeitos de somação relativo em freqüências de fundo mais baixais (Ex.: 2 ciclos $/ 360^{\circ}$ ) e mais altas (Ex.: 48 ciclos/ $360^{\circ}$ ). Estes resultados mostram evidências para interações entre canais de freqüências espaciais angulares em coordenadas polares. Resultados nesta direção já foram descritos na literatura para grade senoidal em coordenadas cartesianas (De Valois, 1977; Tolhurst, 1972; Tolhurst \& Barfield, 1978). Por exemplo, alguns trabalhos com grade senoidal encontraram casos de sensibilidade aumentada para freqüências espaciais à cerca de uma oitava da freqüência da grade de teste (De Valois, 1977; Tolhurst, 1972; Tolhurst \& Barfield, 1978). Esses autores discutiram que isso constitui evidência para interações entre canais de freqüências espaciais. Alguns resultados também mostram interações entre o fundamental e o terceiro harmônico (Pollen \& Ronner, 1982). Apesar de estes resultados serem com padrões cartesianos ao invés de polares, são interessantes na medida em que os nossos resultados para filtros angulares em coordenadas polares também apresentam interação entre canais de freqüência espacial angular de banda estreita. Estes resultados também demonstram que os canais para freqüências espaciais angulares não são totalmente independentes.

Os resultados encontrados para os filtros angulares de 1 e 4 ciclos $/ 360^{\circ}$ com o paradigma de somação de resposta de supralimiar, também, são semelhantes aos resultados encontrados por Simas e colaborados para filtros de freqüências angulares (Simas, 1985; Simas \& Dodwell, 1990; Simas \& cols., 1992) e aos resultados encontrados por Santos e Simas (2002) para freqüências radiais com o mesmo paradigma psicofísico. Ou seja, Simas e colaboradores encontram filtragem ou somação máxima e relativa nas freqüências angulares de teste dos filtros estudados circundadas por inibições. O mesmo foi encontrado por Santos e Simas para os filtros de freqüências radiais de 1 e 4 cpg.

Talvez o pressuposto básico aqui seja a idéia de que, dependendo do filtro espacial (freqüência radial, freqüência angular ou grade senoidal), mecanismos diferentes interdependentes e intradependentes sejam ativados (efeito de inibição, somação e independência dos canais para freqüências espaciais radiais e angulares).

Os resultados nos levam a acreditar que a filtragem realizada pelo sistema visual humano é diferente de filtros mecânicos (ou elétricos) ideais que deixam a quantidade desejada inalterada e reduzem todos os outros valores a zero. Em outras palavras, o processo de filtragem executado pelo sistema visual humano é muito robusto, o que nos leva a crer que a circuitaria visual dispõe de mecanismo para se ajustar a qualquer tipo de padrão espacial, seja ele em coordenadas cartesianas ou polares. Desta forma, o sistema visual pode extrair as características necessárias para reconhecer os detalhes que diferenciam um padrão de outro de acordo com as "suas necessidades". O objetivo principal do sistema visual talvez seja representar o ambiente visual da maneira mais concreta para a realidade na qual o sistema foi adaptado. Há quem acredite que o sistema visual exista para derivar da imagem a informação que nós precisamos e não simplesmente para recriar a imagem projetada na retina (Braddick \& cols., 1978).

Neste contexto, a filtragem espacial realizada pelo sistema visual humano parece ter uma função crucial, pois permite ao sistema visual a lidar seletivamente com uma diversidade muito grande de características simples e complexas de objetos em estágios anteriores que possivelmente são integradas em estágios posteriores, permitindo a reconstrução e interpretação do objeto ou da cena visual, que possivelmente resulta naquilo que denominamos de percepção visual da forma.

Psicologia: Reflexão e Crítica, 2005, 18(1), pp.98-103 
Em síntese, estes resultados são consistentes com a existência de filtros distintos de freqüências angulares em coordenadas polares operando em algum lugar no sistema visual humano.

\section{Referências}

Blakemore, C. \& Campbell, F. C. (1969). Adaptation to spatial stimuli. Journal of Physiology, 200, 11-12.

Braddick, O., Campbell, F. W. \& Atkinson, J. (1978). Channels in vision: Basic aspects. Em R. Held, H. W. Leibowitz, \& H. L. Teuber (Orgs.), Handboob of sensory physiology: Perception (Vol. V; pp. 3-38). New York: Springer-Verlag.

Bruce, C. J., Desimone, R. \& Gross, C. G. (1981). Visual properties of neurons in a polysensory area in superior temporal sulcus of the macaque. Journal of Neurophysiology, 46, 369-384.

Campbell, F. W. \& Robson, F. G. (1968). Application of the Fourier analysis to the visibility of gratings. Journal of Physiology, 197, 551-566.

Dakin, S. C. \& Hess, R. F. (1997). The spatial mechanisms mediating symmetry perception. Vision Research, 37, 2915-2939.

De Valois, K. (1977). Spatial frequency adaptation can enhance contrast sensitivity. Vision Research, 17, 1057-1065.

De Valois, R. L. \& De Valois, K. K. (1988). Spatial vision. New York: Oxford University Press.

Desimone, R. (1991). Face-selective cells in the temporal cortex of monkeys. Journal of Cognitive Neuroscience, 3, 1-8.

Desimone, R. \& Schein, S. J. (1987). Visual properties of neurons in area V4 of macaque: Sensitivity to stimulus form. Journal of Neurophysiology, 57, 835-867.

Gallant, J. L., Connor, C. E., Rakshit, S., Lewis, J. W. \& van Essen, D. C. (1996). Neural responses to polar, hyperbolic, and Cartesian gratings in area V4 of the macaque monkey. Journal of Neurophysiology, 76, 2718-2739.

Gilinski, A. S. (1968). Orientation-specific effects of patterns of adapting light on visual acuity. Journal of the Optical Society of America, 58, 13-18.

Graham, N. (1972). Spatial frequency channels in the human visual system: Effects of luminance and pattern drift rate. Vision Research, 12, 53-63.

Hegde, J. \& van Essen, D. C. (2000). Selectivity for complex shapes in primate visual area V2. Journal of Neuroscience, 20, RC61.

Heywood, C. A., Gadotti, A. \& Cowey, A. (1992). Cortical area V4 and its role in the perception of color. Journal of Neuroscience, 12, 4056-4065.

Maffei, L. \& Fiorentini, A. (1973). The visual cortex as a spatial frequency analyzer. Vision Research, 13, 1255-1267.

Manahilov, V. \& Simpson, W. A. (2001). Energy model for contrast detection: Spatial-frequency and orientation selectivity in grating summation. Vision Research, 41, 1547-1560.

Menees, S. M. (1998). The effect of spatial frequency adaptation on the latency of spatial contrast detection. Vision Research, 38, 3933-3942.

Merigan, W. H. (1996). Basic visual capabilities and shape discrimination after lesions of extrastriate area V4 in macaques. Visual Neuroscience, 13, 51-60.

Olds, E. S. \& Engel, S. A. (1998). Linearity across spatial frequency in object recognition. Vision Research, 38, 2109-2118.

Pollen, D. A. \& Ronner, S. F. (1982). Spatial computation performed by simple and complex cells in the visual cortex of the cat. Vision Research, 22, 101-118.

Sachs, M. B., Nachmias, J. \& Robson, J. G. (1971). Spatial frequency channels in human vision. Journal of the Optical Society of America, 61, 1176-1186.

Santos, N. A. \& Simas, M. L. B. (2001). Percepção e processamento visual da forma: Discutindo modelos teóricos atuais. Psicologia: Reflexão \& Crítica, 14, 151-160.
Santos, N. A. \& Simas, M. L. B. (2002). Percepção e processamento visual da forma em humanos: Filtros de freqüências radiais de 1 e 4 cpg. Psicologia: Reflexão \& Critica, 15, 383-391.

Sekuler, R. (1974). Spatial vision. Annual Review of Psychology, 25, 195-232.

Simas, M. L. B. (1985). Linearity and domain invariance in the visual system (Tese de Doutorado, Queen's University at Kingston, Ontario, Canadá). University Microfilms International, 86, 17940.

Simas, M. L. B. \& Dodwell, P. C. (1990). Angular frequency filtering: A basis for pattern decomposition. Spatial Vision, 5, 59-74.

Simas, M. L. B., Frutuoso, J. T. \& Vieira, F. M. (1992). Inhibitory sidebands in multiple angular filters in the human visual system. Brazilian Journal of Medical and Biological Research, 25, 919-923.

Simas, M. L. S. \& Santos, N. A. (2002a). Narrow-band 1, 2, 3, 4, 8, 16 and 24 cycles/ $360^{\circ}$ angular frequency filters. Brazilian Journal of Medical and Biological Research, 35, 243-253.

Simas, M. L. S. \& Santos, N. A. (2002b). Contrast sensitivity to radial frequencies modulated by Jn and in Bessel profiles. Brazilian Journal of Medical and Biological Research, 35, 1357-1366.

Tolhurst, D. J. (1972). Adaptation to square-wave gratings: Inhibition between spatial frequency channels in the human visual system. Journal of Physiology, 226, 231-248.

Tolhurst, D. J. \& Barfield, L. P. (1978). Interactions between spatial frequency channels. Vision Research, 18, 851-858.

van Essen, D. C., Anderson, C. H. \& Felleman, D. J. (1992). Information processing in the primate visual system: an integrated systems perspective. Science, 255, 419-423.

Vuilleumier, P., Armony, J. L., Driver, J. \& Dolan, R. J. (2003). Distinct spatial frequency sensitivities for processing faces and emotional expressions. Nature Neuroscience, Advance Online Publication (www.nature.com/ natureneuroscience), 1-8.

Wetherill, G. B. \& Levitt, H. (1965). Sequential estimation of points on a psychometric function. The British Journal of Mathematical and Statistical Psychology, 48, 1-10.

Wilkinson, F., Wilson, H. R. \& Habak, C. (1998). Detection and recognition of radial frequency patterns. Vision Research, 38, 3555-3568.

Wilkinson, F., James, T. W., Wilson, H. R., Gati, J. S., Menon, E. S. \& Goodale, M. A. (2000). An fMRI study of the selective activation of human extrastriate form vision areas by radial and concentric gratings. Current Biology, 10, 1455 1458.

Wilson, H. R., Levi, D., Maffei, L., Rovamo, J. \& De Valois, R. (1990). The perception of form: Retina to striate cortex. Em S. W. Spillmann \& J. S. Werner (Orgs.), Visual perception: The neurophysiological foundation (pp. 231-271). New York: Academic Press.

Wilson, H. R. \& Wilkinson, F. (1997). Evolving concepts of spatial channels in vision: From independence to nonlinear interactions. Perception, 26, 939-960.

Wilson, H. R. \& Wilkinson, F. (1998). Detection of global structure in glass patterns: Implications for form vision. Vision Research, 38, 2933-2947.

Wilson, R. W., Wilkinson, F. \& Asaad, W. (1997). Concentric orientation summation in human form vision. Vision Research, 37, 2325-2330.

Young, M. P. (1992). Objective analysis of topological organization of the primate cortical visual system. Nature, 358, 152-155.

Recebido: 05/09/2003

$1^{a}$ revisão: 19/01/2004

Aceite final: $22 / 03 / 2004$

Sobre os autores

Natanael Antonio dos Santos é Mestre e Doutor pela Universidade de São Paulo. É Professor da Universidade Federal da Paraíba.

Renata Maria Toscano Barreto Lyra Nogueira é Psicóloga e Mestranda em Psicologia pela Universidade Federal da Paraíba.

Maria Lúcia de Bustamante Simas é Mestre e Doutora pela Queen's University at Kingston e Pós-doutora pela Universidade Federal do Rio de Janeiro. É Professora da Universidade Federal de Pernambuco. 